\title{
Potencial alelopático das folhas de Pouteria ramiflora (Mart.) RADLK. NA GERMINAÇÃo E DESENVOLVIMENTO INICIAL DE LACTUCA SATIVA L. E SOLANUM LYCOPERSICUM L.
}

\author{
Rosemary Matias ${ }^{1}$, Kelly Cristina lacerda Pereira $^{1}$, Elvia Silvia Rizzi $^{1}$ Clara Anna Araújo Abreu${ }^{1}$, Ana \\ Carolina Rosa ${ }^{1}$, Ademir Kleber Morbeck Oliveira ${ }^{1 *}$
}

\author{
${ }^{1}$ Programa de Pós-Graduação em Meio Ambiente e Desenvolvimento Regional, Universidade Anhanguera-Uniderp \\ *Autorpara correspondência: akmorbeckoliveira@gmail.com
}

Recebido em 23 de abril de 2018. Aceito em 23 de dezembro de 2018. Publicado em 28 de dezembro de 2018.

\begin{abstract}
REsumo - Pouteria ramiflora é uma espécie arbórea indicada para plantio em áreas de preservação permanente. Este trabalho objetivou determinar o potencial alelopático e atividade antioxidante das folhas, assim como realizar sua análise fitoquímica. Os extratos $\mathrm{Ex}_{\mathrm{H} 2 \mathrm{O}}$ (aquoso) e $\mathrm{Ex}_{\mathrm{EtOH}}$ (etanólico) foram submetidos a análise de $\mathrm{pH}$, condutividade elétrica, sólidos solúveis, compostos químicos, atividade antioxidante e testes alelopáticos. Para os bioensaios de germinação de alface e tomate,

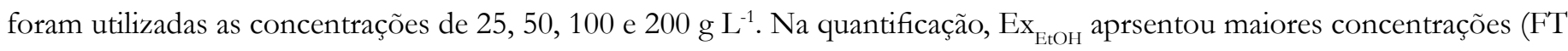
$=275,5 \mathrm{mg} \mathrm{g}^{-1}, \mathrm{~F}=175,6 \mathrm{mg} \mathrm{g}^{-1}$ e $\left.\mathrm{IC}_{50}=367,8 \mathrm{mg} \mathrm{mL}^{-1}\right)$. O processo de germinação das cipselas de alface em câmara de germinação foi afetado apenas por $\mathrm{Ex}_{\mathrm{H} 2 \mathrm{O}}$. Em casa de vegetação, a parte aérea não foi afetada, mas as raízes tiveram menor desenvolvimento. Para as sementes de tomate, a inibição de germinação e aumento no tempo de germinação ocorreu em ambos os extratos, nas maiores concentrações. Em estufa, a parte aérea não sofreu interferência, enquanto o sistema radicular foi afetado. Os resultados obtidos indicam que as folhas da espécie P. ramiflora possuem substâncias com potencial alelopático que interferem negativamente nas espécies testadas.
\end{abstract}

Palavras-chave: Alelopatia, crescimento de plântulas, Sapotaceae.

Allelopathic potential of Pouteria ramiflora (Mart.) Radlk. LEaVes in germination and initial deVelopment OF LACTUCA SATIVA L. AND SOLANUM LYCOPERSICUM L.

AвSTRACT - Pouteria ramiflora is an arboreal species suitable for planting in permanent preservation areas. The work aimed to determine the allelopathic potential and antioxidant activity of the leaves, as well as to perform their phytochemical analysis. The extracts $\mathrm{Ex}_{\mathrm{H} 2 \mathrm{O}}$ (aqueous) and $\mathrm{Ex}_{\mathrm{EtOH}}$ (ethanol) were submitted to $\mathrm{pH}$, electrical conductivity, soluble solids, chemical compounds, antioxidant activity and allelopathic tests. For the germination bioassays of lettuce and tomato, concentrations of 25, 50, 100 and $200 \mathrm{~g} \mathrm{~L}^{-1}$ were used. In the quantification, Ex $\mathrm{EtOH}_{\mathrm{H}}$ presented higher concentrations $\left(\mathrm{FT}=275.5 \mathrm{mg} \mathrm{g}{ }^{-1}, \mathrm{~F}\right.$ $=175.6 \mathrm{mg} \mathrm{g}^{-1}$ and $\left.\mathrm{IC}_{50}=367.8 \mathrm{mg} \mathrm{mL}^{-1}\right)$. The germination process of lettuce cypselae in a germination chamber was only affected by $\mathrm{Ex}_{\mathrm{H} 2 \mathrm{O}}$. In the greenhouse, the aerial part was not affected, but the roots presented smaller development. For tomato seeds, inhibition and increase in the germination rate occurred in both extracts at the highest concentrations. In the greenhouse, the aerial part did not suffer interference, while the root system was affected. The results indicate that the leaves of the species $P$. ramiflora carry out substances with allelopathic potential that negatively interfere on the tested species.

Keywords: Allelopathy, Seedling growth, Sapotaceae. 
Potencial alelopático de las hojas de Pouteria Ramiflora (Mart.) Radlk. en la germinación y desarkollo inicial DE LACTUCA SATIVA L. Y SOLANUM LYCOPERSICUM L.

RESUMEN - Pouteria ramiflora es una especie arbórea indicada para la siembra en áreas de preservación permanente. Este trabajo tuvo como objetivo determinar el potencial alelopático y actividad antioxidante de las hojas y realizar su análisis fitoquímico. Los extractos $\mathrm{Ex}_{\mathrm{H} 2 \mathrm{O}}$ (acuoso) y $\mathrm{Ex}_{\mathrm{EtOH}}$ (etanólico) fueron sometidos a análisis de $\mathrm{pH}$, conductividad eléctrica, sólidos solubles, compuestos químicos, actividad antioxidante y pruebas alelopáticas. Para los bioensayos de germinación de lechuga y tomate, se han utilizado concentraciones de 25, 50, 100 y $200 \mathrm{~g} \mathrm{~L}^{-1}$. En la cuantificación, Ex $\mathrm{EtOH}_{\mathrm{Se}}$ ha presentado mayores concentraciones ( $\mathrm{FT}=275,5 \mathrm{mg} \mathrm{g}^{-1}, \mathrm{~F}=175,6 \mathrm{mg} \mathrm{g}^{-1}$ y IC50 $\left.=367,8 \mathrm{mg} \mathrm{mL}^{-1}\right)$. El proceso germinativo de las cipselas de lechuga en la cámara de germinación fue afectado solamente por $\mathrm{Ex}_{\mathrm{H} 2 \mathrm{O}}$. En invernadero, la parte aérea no fue afectada, sin embargo, el desarrollo de las raíces fue menor. La inhibición germinativa y el aumento en el tiempo de germinación de las semillas de tomate, sucedió en ambos extractos y en las mayores concentraciones. En invernadero, la parte aérea no ha sufrido interferencia, mientras que el sistema radicular fue afectado. Estos resultados obtenidos indican que las hojas de la especie $P$. ramiflora poseen substancias con potencial alelopático que interfieren negativamente en las especies evaluadas.

Palabras Clave: Alelopatía, crecimiento de plántulas, Sapotaceae.

\section{INTRODUÇÃO}

Um dos fatores que podem limitar o desenvolvimento das atividades agrícolas é a alta incidência de espécies invasoras (Zeng et al. 2010). O uso de herbicidas para controlar estas plantas, em grande quantidade, pode causar toxicidade do produto ao ambiente, além de selecionar a flora e levar ao aparecimento de resistência em alguns grupos (Nemoto 2005).

Visando substituir os químicos sintéticos, os herbicidas naturais são vistos como uma ação complementar para o controle das espécies invasoras, motivando pesquisas voltadas à alelopatia, buscando a sustentabilidade na produção e conservação da vegetação natural, com menor impacto ambiental (Zeng et al. 2010).

Algumas plantas possuem estratégias de defesa contra competição de outras plantas através da liberação de substâncias químicas, que podem agir de forma direta (interferência no crescimento e metabolismo) ou indireta (interferência na biodiversidade, por exemplo) (Willis 2007; Zeng et al. 2010).

Essas substâncias normalmente são encontradas em diversas partes da planta e são liberadas por lixiviação dos tecidos, exsudação radicular, decomposição dos tecidos e volatilização (Rice 1984). Dentre os compostos secundários que apresentam essas interferências estão os taninos, glicosídeos cianogênicos, alcaloides, sesquiterpenos e flavonoides, entre outros compostos (Willis 2007).

Com isso, pesquisas voltadas à alelopatia apresentam grande importância e o meio mais comum de determinar o potencial alelopático que uma planta tem sobre outra é através de estudos dos efeitos dos extratos da planta desejada sobre sementes ou plântulas de espécies cultivadas (Rice 1984). As interações frequentemente observadas são: inibição da percentagem de germinação e redução da velocidade da germinação, além do efeito negativo sobre o crescimento inicial das plântulas (Pedro et al. 2006).

O desenvolvimento inicial da plântula é mais sensível aos efeitos das substâncias químicas, enquanto que na germinação esses efeitos são mais discretos (Ferreira e Áquila 2000). Além disto, um composto que é tóxico para uma espécie pode não ser para outra (Willis 2007).

Por apresentar maior sensibilidade aos metabólitos secundários, algumas espécies acabam sendo utilizadas como bioindicadoras. O tomate (Solanum lycopersicum L.) e a alface (Lactuca sativa L.) apresentam, além da sensibilidade, germinação rápida e uniforme, sendo utilizadas em bioensaios como espécies-alvo (Ferreira e Áquila 2000).

Em alguns biomas do Brasil, como o Cerrado, os estudos alelopáticos ainda são incipientes e uma das famílias encontradas neste ambiente, Sapotaceae, é comumente utilizada na medicina popular; possui utilização para o 
tratamento de febres e resfriados, malária e diabetes, entre outros usos, possuindo uma diversidade de metabólitos secundários, tais como alcaloides, flavonoides, terpenoides e benzenoides, por exemplo (Montenegro et al. 2006).

Nesta família, encontra-se Pouteria ramiflora (Mart.) Radlk., conhecida popularmente por curriola, brasa-viva ou fruta-de-viado (Lorenzi 2002). Por apresentar crescimento moderado e adaptação a lugares abertos, é indicada para plantio em áreas de preservação permanente. Além disto, os extratos aquosos e etanólicos desta espécie apresentam efeitos alelopáticos, interferindo no crescimento de plântulas de alface e tomate, fator provavelmente associado à presença de esteroides e triterpenos, além de outros compostos, presentes nos extratos (Silva et al. 2006; Condessa et al. 2013; Fernandes et al. 2014; Oliveira et al. 2014a).

Desta maneira, a indicação da espécie para o uso em plantios mistos em áreas degradadas e seu potencial de utilização justifica a necessidade de estudos para verificar sua capacidade de interferir no crescimento de outros vegetais através da alelopatia. Apesar de já existirem outros trabalhos similares, a relação com metabólitos presentes nas folhas ainda não foi analisada.

Neste contexto, o presente trabalho objetivou avaliar o potencial alelopático e atividade antioxidante das folhas de P. ramiflora, assim como realizar sua análise fitoquímica.

\section{Material e Métodos}

Coleta - As folhas de P. ramiflora foram coletadas manualmente em matas da região do Taboco, município de

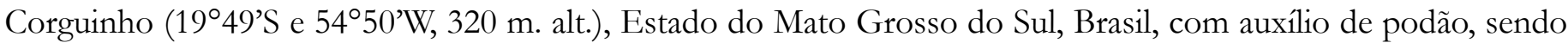
acondicionadas em sacos de polietileno estéreis e levadas para Laboratório de Pesquisa em Sistemas Ambientais e Biodiversidade - LabPSAB, Campo Grande, Mato Grosso do Sul.

Após a triagem do material, com a eliminação das folhas deterioradas ou danificadas, as demais foram secas $\left( \pm 27^{\circ} \mathrm{C}\right)$ por 72 horas e trituradas em moinho industrial. O material foi armazenado em frasco de vidro, vedado, rotulado e guardado em geladeira (para evitar o efeito negativo da temperatura e luminosidade nos metabolitos) até a preparação dos extratos.

Preparação dos extratos - Os extratos, aquoso $\left(\mathrm{Ex}_{\mathrm{H} 2 \mathrm{O}}\right)$ e etanólico $\left(\mathrm{Ex}_{\mathrm{EtOH}}\right)$ a $20 \%\left(200 \mathrm{~g} \mathrm{~L}^{-1}\right)$, foram preparados, separadamente, via turbólise por 10 minutos, e em sequência, transferidos para béqueres de $2 \mathrm{~L}$ e armazenados em geladeira $\left( \pm 4^{\circ} \mathrm{C}\right)$ por 24 horas, na ausência de luz. Após este período, foram submetidos a banho de ultra-som por duas horas e mantidos em repouso na ausência de luz por mais 48 horas, em geladeira, sendo posteriormente filtrados (funil de vidro e algodão) em balão volumétrico de $200 \mathrm{~mL}$. A partir do filtrado bruto (20\%) foi feita a diluição em concentrações menores de 25, 50 e 100,0 $\mathrm{g} \mathrm{L}^{-1}$ (Oliveira et al. 2011).

Fitoquímica - Para os ensaios de detecção dos constituintes químicos, os extratos $\mathrm{Ex}_{\mathrm{H} 2 \mathrm{O}}$ e $\mathrm{Ex}_{\mathrm{EtOH}}(200 \mathrm{~g}$ $\left.\mathrm{L}^{-1}\right)$ foram submetidos à análise fitoquímica via úmida, por meio de reações de precipitação e/ou mudança de cor (Quadro 1). Já a análise confirmatória de saponinas foi determinada utilizando-se $1 \mathrm{~g}$ do extrato seco, para estimar o índice de espuma (índice afrosimétrico) (Matos 2010). 
Quadro 1. Testes padrões utilizados para reconhecimento de metabólitos secundários das folhas de Pouteria ramiflora

\begin{tabular}{|l|l|}
\hline \multicolumn{1}{|c|}{ Metabólitos secundários } & \multicolumn{1}{c|}{ Métodos de análise } \\
\hline Compostos fenólicos & $\begin{array}{l}\text { Reações de precipitação e/ou de mudança de cor: cloreto férrico a 2\%; acetato de } \\
\text { chumbo a } 10 \% \text { e acetato de cobre a 4\%. }\end{array}$ \\
\hline Taninos & $\begin{array}{l}\text { Reações de precipitação e/ou de mudança de cor: reação de cloreto férrico 1\% e } \\
\text { precipitação da proteína. }\end{array}$ \\
\hline Flavonoides & Reações de mudança de cor: reação de Shinoda (Mg/HCl) e de Taubock. \\
\hline Antocianinas & Reações de mudança de cor: reações ácido/base. \\
\hline Cumarinas & Reação de mudança de cor: reação com KOH e análise à luz ultravioleta \\
\hline Antraquinonas livres & Reação de mudaça de cor: reação de Borntrager \\
\hline Glicosídeos antraquinônicos & Reação de mudança de cor: reação de Borntrager com hidrólise prévia. \\
\hline Esteroides & Reação de mudança de cor: reação de Liebermann Burchard. \\
\hline Triterpenos livres & Reação de mudaça de cor: reção de Liebermann Burchard. \\
\hline Alcaloides & $\begin{array}{l}\text { Reações de precipitação e/ou reações de mudança de cor: Dragendorff, Hager, Mayer } \\
\text { e Bertrand. }\end{array}$ \\
\hline Glicosídeos cianogênicos & Reações de mudança de cor: Teste do papel de picrato de sódio. \\
\hline Glicosídeos carditônicos & $\begin{array}{l}\text { Reações de mudança de cor: reação de Kedde (reações para detectar o anel lactônico } \\
\text { insaturado); reação de K Keller-Killiani (reações determinar os desoxi-açúcares). }\end{array}$ \\
\hline Saponinas & Reação de Lieberman-Buchard e de formação de espuma. \\
\hline Açúcares redutores & Reação de precipitação e/ou de mudança de cor: Teste de Benedict. \\
\hline
\end{tabular}

As análises foram executadas em triplicatas e comparadas com a amostra controle $\left(\mathrm{Ex}_{\mathrm{H} 2 \mathrm{O}}\right.$ e $\mathrm{Ex}_{\mathrm{EtOH}}$ a $\left.20 \%\right)$. As leituras dos resultados foram feitas através da observação da alteração de cor e precipitação do filtrado (Costa 2002). Para cada teste, as intensidades foram classificadas como sendo parcial ( \pm ), baixa $(+)$, moderada $(++)$ e alta intensidade $(+++)$, além de negativa $(-)$. Os testes com formação de precipitado (compostos fenólicos, taninos e açúcares redutores) foram realizados em tubos de graduação (No.8080, Pyrex®) e considerados como parcial (menos de 0,2 cm), baixo $(0,2$ a $0,5 \mathrm{~cm})$, moderada $(0,5$ a $0,7 \mathrm{~cm})$ e alta intensidade $(0,7$ a 1,0 cm) (Fountoura et al. 2015).

Os extratos foram usados para quantificar os fenóis totais (FT), determinados pelo Método Folin-Ciocalteu, com ácido gálico (10 a $350 \mathrm{mg} \mathrm{mL}^{-1}$ ) como padrão (Sousa et al. 2007) e flavonoides (F), avaliados pelo método cloreto de alumínio e como padrão, quercetina, para construir a curva de calibração (Peixoto Sobrinho et al. 2008). Também submetidos às análises de $\mathrm{pH}$, condutividade elétrica e concentração de sólidos solúveis, determinada utilizando um refratômetro digital, com resultados expressos em graus Brix corrigidos para $20^{\circ} \mathrm{C}$.

Atividade antioxidante - Os extratos a $20 \mathrm{~g} \mathrm{~L}^{-1}$ foram submetidos a análise da atividade antioxidante, empregando-se o método DPPH (2,2-difenil-1-picril-hidrazil). As amostras nas concentrações de 25, 50, 100, 200 e $250 \mu \mathrm{g} \mathrm{mL}^{-1}$ foram adicionadas à $2 \mathrm{~mL}$ de uma solução de DPPH em metanol (24 mg $100 \mathrm{~mL}^{-1}$ de metanol). Após 30 min, a absorbância foi determinada em espectrofotômetro, a $515 \mathrm{~nm}$. A solução de DPPH em metanol foi utilizada como controle negativo e tendo como controle positivo, BHT (butilhidroxitolueno) nas mesmas concentrações das amostras (Thaipong et al. 2006). A percentagem de inibição (\% I) foi calculada segundo Sousa et al. (2007), onde:

IA $(\%)=1$ - (Abs amostra/Abs controle) x 100, onde, Abs amostra corresponde a absorbância da amostra e Abs controle, a absorbância do controle totalmente oxidado.

Bioensaios de germinação - Foram utilizados $5 \mathrm{~mL}_{\text {de }} \mathrm{Ex}_{\mathrm{H} 2 \mathrm{O}} \mathrm{e} \mathrm{Ex}_{\mathrm{EtOH}}$ a $20 \mathrm{~g} \mathrm{~L}^{-1}$ nas diferentes concentrações, além do controle água destilada e controle etanol (controle etanol: $5 \mathrm{~mL}$ de etanol na placa, deixada na bancada para evaporação, após o qual foram colocados $5 \mathrm{~mL}$ de água destilada), sobre duas folhas de papel Germitest ${ }^{\circledR}$ em placas de Petri de $7 \mathrm{~cm}$ de diâmetro (quatro repetições com 25 sementes de L. sativa, variedade "maravilha quatro estações" e L. esculentum, "Santa Clara”). As placas não foram umedecidas novamente durante o período do experimento (7 dias). A observação das sementes germinadas foi feita a cada 24 horas, onde foram consideradas 
germinadas apenas as sementes que apresentassem $2 \mathrm{~mm}$ de protrusão de raiz primária. Os procedimentos seguiram padrões de Oliveira et al. (2014ab).

Bioensaios de crescimento - Foram utilizados $10 \mathrm{~mL}$ dos extratos nas mesmas concentrações usadas nos bioensaios de germinação, sobre duas folhas de papel Germitest ${ }^{\circledR}$ em caixas plásticas transparentes $(11$ x 11 x 3,5 $\mathrm{cm})$ lacradas com papel filme, com 10 sementes pré-germinadas com $2 \mathrm{~mm}$ de sistema radicular para o teste de crescimento. A avaliação foi feita 10 dias após a semeadura e a medição da raiz primária, feita do colo da plântula até o ápice meristemático do sistema radicular $(\mathrm{mm})$ e da parte aérea $(\mathrm{mm})$, do colo da planta até o ápice, com auxílio de paquímetro digital. Os bioensaios foram realizados em câmaras de germinação nas temperaturas de $20{ }^{\circ} \mathrm{C}$ para alface e $25^{\circ} \mathrm{C}$ para tomate, com fotoperíodo de 12 horas de luz branca. Os procedimentos seguiram padrões de Oliveira et al. (2014ab).

Para a análise dos resultados de germinação, foram calculados a percentagem de germinação (G\%), o tempo médio de germinação (TMG) (Vieira e Carvalho 1994) e o índice de velocidade de germinação (IVG) (Maguire 1962), através da contagem diária de sementes germinadas. O delineamento experimental foi inteiramente casualizado.

Bioensaios de casa de vegetação - Realizados em bandejas de isopor, nas concentrações de 100 e $200 \mathrm{~g}$ $\mathrm{L}^{-1}$ (pó da planta misturada ao substrato vermiculita) e o controle feito apenas com vermiculita, uma vez que este substrato apresenta alta capacidade de retenção de água e ar, além de ser um material leve, puro, não abrasivo, inodoro, de pH essencialmente neutro e inerte (Martins 2009). A vermiculita pode garantir que nenhum fator externo, tais como pH, matéria orgânica, argila do solo ou microrganismos, mascare a ação dos metabólitos.

Após a mistura, o material foi umedecido com água destilada ficando em repouso por um dia. Para semeadura nas células, foram utilizadas 60 sementes de cada espécie para cada tratamento, sendo uma semente por célula.

O processo de emergência da parte aérea foi observado diariamente por 10 dias e após esse período, realizouse a medição do sistema radicular e parte aérea; também foi avaliado o índice de velocidade de emergência (IVE) (Maguire 1962), com delineamento experimental inteiramente casualizado.

Análise estatística - Para comparação entre os tratamentos, foi utilizada a estatística descritiva através da comparação de intervalos de confiança das médias (Costa Neto 2002; Ferreira 2005) por meio do teste t (Snedecor e Cochram 1973), realizada através do programa Assistat em nível de 5\% ( $<<0,05)$.

\section{Resultados e Discussão}

A análise fitoquímica de $\mathrm{Ex}_{\mathrm{H} 2 \mathrm{O}}$ indicou seis classes de metabólitos secundários, porém com menor intensidade em relação a $\mathrm{Ex}_{\mathrm{EtOH}}$, que apresentou sete classes, com predominância dos compostos fenólicos, flavonoides, triterpenos e esteroides. A quantificação de fenóis totais de $\mathrm{Ex}_{\mathrm{EtOH}}$ das folhas foi superior e estatisticamente diferente da média do $\mathrm{Ex}_{\mathrm{H} 2 \mathrm{O}^{\circ}}$ Os flavonoides totais seguiram o mesmo perfil dos fenóis totais, com valores de $\mathrm{Ex}_{\mathrm{EtOH}}$ superiores e estatisticamente diferente da média de $\mathrm{Ex}_{\mathrm{H} 2 \mathrm{O}}$ (Tabela 1). A presença destas classes de metabólitos secundários justifica a atividade antioxidante do extrato $\operatorname{Ex}_{\mathrm{H} 2 \mathrm{O}}\left(153,2 \pm 0,8 \mathrm{mg} \mathrm{L}^{-1}\right)$ e $\operatorname{Ex}_{\mathrm{EtOH}}\left(367,8 \pm 1,2 \mathrm{mg} \mathrm{L}^{-1}\right)$ (Tabela 1).

Entre os metabólitos testados, antocianinas, antraquinonas livres, alcaloides e glicosídeos antraquinônicos, cardiotônicos e cianogênios não foram detectados nas amostras.

Dentre a diversidade de constituintes químicos da família Sapotaceae, são comuns os triterpenos e flavonoides, sendo que os últimos são descritos como marcadores quimiotaxonômicos para as espécies de Pouteria, ocorrendo com maior frequência nas folhas (Silva et al. 2009). Neste estudo, dos fitoconstituintes investigados, os compostos fenólicos, taninos e flavonoides foram predominantes nos extratos $\mathrm{Ex}_{\mathrm{H} 2 \mathrm{O}}$ e $\mathrm{Ex}_{\mathrm{EtOH}}$ de P. ramiflora, confirmando a afirmação acima.

Os compostos fenólicos correspondem a um grupo de aleloquímicos apontados como os de maior efeito alelopático e a essa classe corresponde aos fenóis simples e flavonoides até estruturas mais complexas, como os 
taninos, considerados potentes inibidores da germinação de sementes (Rice 1984). De acordo com Fujii e Hiradate (2007), taninos diminuem a formação de lignina, contribuindo para a redução no alongamento radicular, além de bloquear a respiração mitocondrial, prejudicando o processo de germinação, como observado para as espécies testadas.

O fato de um grupo de compostos afetar a resposta fisiológica das sementes, como a respiração, é indicativo da capacidade antioxidante de extratos vegetais, geralmente relacionada à presença de compostos fenólicos e pontualmente aos flavonoides, ambos encontrados nos extratos testados. Porém $\mathrm{Ex}_{\mathrm{EtOH}}$ apresentou maior atividade, demonstrando maior concentração destes metabólitos. De acordo com Silva et al. (2009), o potencial antioxidante dos extratos é uma das características biológicas das espécies de Pouteria, fator atribuído a presença de compostos fenólicos, normalmente nos extratos e frações mais polares, como $\mathrm{Ex}_{\mathrm{EtOH}}$.

Pergo e Ishii-Iwamoto (2011) também relatam em estudos na área de alelopatia a presença de agentes antioxidantes, porém com atividade relacionada às proteínas de defesa da planta, tais como as enzimas catalase, peroxidase e superóxido dismutase. No entanto, os mesmos autores, em testes "in vitro", descrevem que o estresse oxidativo ou "estresse aleloquímico" tem a capacidade de desencadear os mecanismos de defesa na espécie-alvo, influenciando, por exemplo, na germinação e crescimento de outras espécies.

Os resultados obtidos indicaram que ocorreu efeito alelopático sobre a germinação, IVG e TMG de sementes de alface e tomate (Tabelas 2 e 3) e no comprimento das raízes e caules de plântulas de tomate e alface, em câmara de germinação e casa de vegetação (Tabelas 4 e 5). De acordo com essas informações, pode-se inferir que a atividade antioxidante foi um dos fatores a influenciar no efeito alelopático dos extratos, ocasionando estresse oxidativo e afetando o processo de germinação das sementes de alface (L. sativa) e tomate (L. esculentum) e a formação de plântulas.

Este efeito pode ser atribuído aos flavonoides e compostos fenólicos, que foram tóxicos para as células, afetando seu crescimento. Este fato possivelmente ocorreu pela capacidade destes metabólitos atuarem como agente hiperpolarizador de membrana, alterando os mecanismos das bombas de $\mathrm{H}^{+}$(Parvez et al. 2004). Nicollier e Thompson (1982) também indicam que estes compostos podem alterar a homeostase enzimática celular e consequentemente, inibir e/ou atrasar a germinação das sementes, fato observado nos testes realizados.

As cumarinas, detectadas nos dois extratos em baixa intensidade, também são apontadas como inibidoras potentes, tanto da germinação como do crescimento de plantas (Willis 2007), devido sua capacidade de bloquear a mitose e diminuir a entrada de água na célula, entre outras ações (Abenalovi et al. 2006).

Já as saponinas, também encontradas, porém apenas no extrato aquoso, se dividem em dois grupos: esteroides e triterpenoides, encontrados em maior intensidade no extrato etanólico, parte com caráter lipofílico e parte hidrofílica. Em meio aquoso, as saponinas são afrogênicas e, sendo assim, possuem elevada solubilidade em água; já nos solventes orgânicos, como álcool, possuem baixa solubilidade (Simões et al. 2004).

Assim, a maior atividade do extrato aquoso em relação ao extrato etanólico nas sementes pode ser justificada pela presença das saponinas, por sua solubilidade em água e capacidade emulsificante, que causam efeitos alelopáticos. Segundo Weir et al. (2004), as saponinas têm capacidade de interagir com as membranas celulares, modificando sua permeabilidade e desorganizando-a, além de causar desequilíbrios hormonais e atuar diretamente no processo fotossintético.

Neste trabalho, foram encontrados valores de $\mathrm{pH}$ 4,8 em $\mathrm{Ex}_{\mathrm{H} 2 \mathrm{O}}$ e 5,5, $\mathrm{Ex}_{\mathrm{EtOH}}$; para condutividade elétrica, extratos $\mathrm{Ex}_{\mathrm{H} 2 \mathrm{O}}, \mathrm{CE}=132,0 \mu \mathrm{S} \mathrm{cm}{ }^{-1}$ e $\mathrm{Ex}_{\mathrm{EtOH}}, \mathrm{CE}=94,0 \mu \mathrm{S} \mathrm{cm}^{-1}$; os sólidos dissolvidos (teor aproximado de açúcar e sólidos totais solúveis) encontrados, medidos em grau Brix, revelaram que $\mathrm{Ex}_{\mathrm{EtOH}}$ possuia grau Brix zero e, $\mathrm{Ex}_{\mathrm{H} 2 \mathrm{O}}$, tendendo a zero (Tabela 1). 
Tabela 1. Composição química, teor de fenóis (FT), flavonoides totais (F), índice de atividade antioxidante $\left(\mathrm{IC}_{50}\right)$, $\mathrm{pH}$, condutividade elétrica (CE) e sólidos solúveis (SS), extrato aquoso $\left(\mathrm{Ex}_{\mathrm{H} 2 \mathrm{O}}\right)$ e etanólico $\left(\mathrm{Ex}_{\mathrm{E}+\mathrm{OH}}\right)$, folhas de Pouteria ramiflora

\begin{tabular}{|c|c|c|}
\hline \multirow{2}{*}{ Metabólitos Secundários } & \multicolumn{2}{|c|}{ Extrato } \\
\hline & $\mathbf{E x}_{\mathrm{H} 2 \mathrm{O}}$ & $\mathbf{E x}_{\mathrm{EtOH}}$ \\
\hline Compostos fenólicos & ++ & +++ \\
\hline Taninos & ++ & ++ \\
\hline Flavonoides & ++ & +++ \\
\hline Cumarinas & + & + \\
\hline Triterpenos & - & +++ \\
\hline Esteroides & - & +++ \\
\hline Açúcares redutores & ++ & + \\
\hline Saponinas* & + & - \\
\hline FT (mg de equivalentes de ácido gálico/g) & $87,0 \pm 1,1$ & $275,5 \pm 0,2$ \\
\hline $\mathrm{F}$ (mg de equivalentes de quercetina/g) & $22,4 \pm 1,3$ & $175,6 \pm 1,0$ \\
\hline $\mathrm{IC}_{50}\left(\mathrm{mg} \mathrm{mL}^{-1}\right)$ & $153,2 \pm 0,8$ & $367,8 \pm 1,2$ \\
\hline $\mathrm{pH}$ & 4,8 & 5,5 \\
\hline $\mathrm{CE}\left(\mu \mathrm{S} \mathrm{cm} \mathrm{cm}^{-1}\right)$ & 132,0 & 94,0 \\
\hline SS $\left({ }^{\circ}\right.$ Brix a $\left.20^{\circ} \mathrm{C}\right)$ & 0,3 & 0,0 \\
\hline
\end{tabular}

*Análise em extrato seco. Menor intensidade (+), média (++), maior (+++) e, negativo (-). $\mathrm{IC}_{50}=$ Inibição de $50 \%$ da concentração inicial.

Os valores de $\mathrm{pH}$ encontrados são considerados dentro dos limites toleráveis pelas sementes, não representando um fator de interferência nos processos de germinação e formação de plântulas. De acordo com Ferreira e Áquila (2000), valores extremos de acidez e basicidade podem encobrir o efeito das substâncias químicas, o que não ocorreu. Rice (1984) relata que a alface mostra pouca sensibilidade para diferentes escalas de $\mathrm{pH}$ e somente os extremos de acidez ou alcalinidade, onde o pH é de 3,0 ou menos (muito ácido) ou superior ou igual a 9,0 ou 11 (extremamente alcalino) pode afetar a germinação.

Os resultados encontrados de $\mathrm{pH}$, entre 4,8 e 5,5, são similares aos citados por Oliveira et al. (2014b) com casca da mesma espécie, com pH do extrato etanólico entre 5,6 e 6,6 e aquoso, 4,8 e 5,5. Os autores citam que os valores observados não interferiram no processo de germinação em todas as concentrações e extratos testados, como observado neste trabalho.

Estes resultados indicam que os extratos de P. ramiflora possuem características similares de $\mathrm{pH}$, independente do órgão avaliado, sendo mais ácido no extrato aquoso. Porém esta maior acidez não interfere nos processos de germinação e crescimento das plântulas, pois as espécies-alvo conseguem se desenvolver em uma ampla faixa de $\mathrm{pH}$, conforme relatado por Rice (1984).

Dados obtidos com outra espécie arbórea do Cerrado, Palicourea rigida Kunth., coletada no Estado de Mato Grosso do Sul, em ensaios de alelopatia, também indicaram padrão similar de $\mathrm{pH}$, com extrato aquoso entre 4,0 e 4,7 \pm 0,5, e extrato etanólico, entre 4,8 e 5,3 \pm 0,5 (Oliveira et al. 2014b). Os resultados para estas espécies ( $P$. rigida e $P$. ramiflora) indicaram novamente que os extratos aquosos possuem maior acidez, porém dentro da faixa de tolerância das espécies-alvo, não tendo inferido negativamente na germinação e crescimento das plântulas.

Outro fator que poderia interferir nos resultados obtidos, ou seja, a atividade alelopática sobre a germinação e formação de plântulas, seria a condutividade elétrica dos extratos; porém a mesma não apresentou valores que afetassem o processo de germinação. De acordo com Dias e Marcos Filho (1995), em extratos vegetais são encontrados além dos metabólitos secundários, íons inorgânicos, açúcares, aminoácidos, enzimas, entre outros, que podem alterar o potencial osmótico das soluções, interferindo negativamente na germinação. Souza et al. (1999) indicaram que uma condutividade inferior a $200 \mu \mathrm{S} \mathrm{cm}^{-1}$ não influenciou de forma negativa a germinação 
de sementes de alface e cenoura, demonstrando que apenas valores mais elevados poderiam afetar o processo germinativo.

Levando-se em consideração que na quantificação de sólidos dissolvidos encontrados nos extratos, a presença de açúcares é um demonstrativo da potencialidade osmótica da solução, um grau Brix pequeno e uma condutividade inferior a $200 \mu \mathrm{Scm}^{-1}$ são indicativos de um potencial osmótico baixo. Desta maneira, estes resultados não afetariam negativamente o processo germinativo e o crescimento de espécies submetidas aos diferentes extratos.

Os valores obtidos para estas características, em relação a espécie testada neste trabalho, P. ramiflora, são parcialmente similares aos citados por Oliveira et al. (2014b) com P. rigida, onde foram encontrados valores de condutividade elétrica entre 25 e $173 \mu \mathrm{S} \mathrm{cm}^{-1}$ (extrato aquoso) e 0,7 e $1,3 \mu \mathrm{S} \mathrm{cm}$ (extrato etanólico) e sólidos solúveis, para ambos os extratos, 0,8 . Os autores consideram que estes resultados não interferiram negativamente nos processos germinativos das espécies-alvo.

Os dados obtidos para $P$. ramiflora demonstram que os extratos interferem negativamente no processo germinativo das espécies; porém as sementes de tomate demonstraram maior suscetibilidade para ambos os extratos, nas maiores concentrações, enquanto que as de alface foram afetadas apenas pelo extrato aquoso. A maior sensibilidade de uma espécie a um determinado tipo de metabólico pode estar relacionada a seu táxon, como família e gênero, por exemplo, pois cada grupo pode possuir processos bioquímicos próprios e desta maneira, reagir de maneira diferente as substâncias encontradas (Ferreira e Aquila 2000).

Este mesmo padrão de ação foi encontrado por Silva et al. (2006) e Fernandes et al. (2014), com ação alelopática de extratos testados afetando mais fortemente a germinação e vigor das sementes de tomate, em relação a alface ou outras espécies testadas.

De acordo com Souza et al. (2005), as sementes de alface (L. sativa) possuem alta sensibilidade ao estresse ambiental. Desta maneira, seria esperado que a alface fosse influenciada mais fortemente, o que não ocorreu no experimento realizado com os extratos de P. ramiflora, com sementes de tomate demonstrando maior sensibilidade. Estes resultados indicam a importância na utilização de diferentes espécies-alvo para testes de alelopatia.

O padrão de interferência dos metabólitos secundários pode ser variável, na dependência da espécie testada. Para algumas espécies, como nabo (Raphanus raphanistrum L.), Wandscheer e Pastorini (2008), testando extratos de folhas e raízes, observaram que nas concentrações 5 e 10\%, os extratos influenciaram na velocidade de germinação de sementes. Por outro lado, Capobianco et al. (2009) avaliando cutia (Joanesia princeps Vell.) e carvalhinho (Casearia sylvestris Sw.), demonstraram que concentrações de 10 e 30\% reduziram o IVG; entretanto em maiores concentrações, 70 a 100\%, não ocorreu interferência.

Porém o padrão mais encontrado é o similar ao deste trabalho com P. ramiflora, com as maiores concentrações dos extratos tendendo a afetar negativamente as sementes. Sausen et al. (2009), em trabalho com cerejeira-do-mato (Eugenia involucrata DC.) e goiabeira-serrana (Acca sellowiana (O. Berg) Burret.), demonstraram a mesma sequência, onde as concentrações mais diluídas ( 25 e $50 \%$ ), não apresentaram interferência significativa e apenas as maiores, 75 e $100 \%$, interferiram no processo germinativo.

Os dados de germinação (Tabela 2), para sementes de alface, indicaram que $\mathrm{Ex}_{\mathrm{EtOH}}$ não afetou a germinação e o tempo de germinação, em nenhuma das concentrações testadas. Já $\mathrm{Ex}_{\mathrm{H} 2 \mathrm{O}}$ afetou a percentagem de germinação e o vigor das sementes, interferindo na velocidade de germinação a partir das concentrações de 25,0 ou 50,0 $\mathrm{g} \mathrm{L}^{-1}$, comparados com o grupo controle. 
Tabela 2. Germinação (\%), índice de velocidade de germinação (IVG) e tempo médio de germinação (TMG) de sementes de alface, $\mathrm{Ex}_{\mathrm{H} 2 \mathrm{O}}$ e $\mathrm{Ex}_{\mathrm{EtOH}}$, folhas de Pouteria ramiflora

\begin{tabular}{ccccccc}
\hline & \multicolumn{2}{c}{ Germinação $(\%)$} & \multicolumn{2}{c}{ TVG } & \multicolumn{2}{c}{ TMG } \\
\hline Extrato $\left(\mathrm{g} \mathrm{L}^{-1}\right)$ & $\mathrm{Ex}_{\mathrm{H} 2 \mathrm{O}}$ & $\mathrm{Ex}_{\mathrm{EtOH}}$ & $\mathrm{Ex}_{\mathrm{H} 2 \mathrm{O}}$ & $\mathrm{Ex}_{\mathrm{EtOH}}$ & $\mathrm{Ex}_{\mathrm{H} 2 \mathrm{O}}$ & $\mathrm{Ex}_{\mathrm{EtOH}}$ \\
\hline 0 & $98 \mathrm{a}$ & $100 \mathrm{a}$ & $15,9 \mathrm{a}$ & $38,9 \mathrm{a}$ & $0,89 \mathrm{~d}$ & $0,43 \mathrm{a}$ \\
25,0 & $98 \mathrm{a}$ & $100 \mathrm{a}$ & $17,5 \mathrm{a}$ & $39,6 \mathrm{a}$ & $0,34 \mathrm{a}$ & $0,41 \mathrm{a}$ \\
50,0 & $91 \mathrm{~b}$ & $98 \mathrm{a}$ & $13,9 \mathrm{~b}$ & $38,5 \mathrm{a}$ & $0,46 \mathrm{~b}$ & $0,42 \mathrm{a}$ \\
100,0 & $83 \mathrm{~b}$ & $100 \mathrm{a}$ & $10,3 \mathrm{c}$ & $38,7 \mathrm{a}$ & $0,63 \mathrm{c}$ & $0,43 \mathrm{a}$ \\
200,0 & $62 \mathrm{c}$ & $100 \mathrm{a}$ & $3,5 \mathrm{~d}$ & $38,4 \mathrm{a}$ & $1,2 \mathrm{e}$ & $0,44 \mathrm{a}$ \\
\hline
\end{tabular}

* Médias seguidas pelas mesmas letras dentro das colunas não diferem entre si, pelo teste t (5\% de probabilidade).

Para as sementes de tomate (Tabela 3), ambos os extratos, Ex $\mathrm{H}_{2 \mathrm{O}}$ e $\mathrm{Ex}_{\mathrm{EtOH}}$, influenciaram na percentagem de germinação, nas maiores concentrações, 100 e $200 \mathrm{gL}^{-1}$ e no vigor das sementes, diminuindo a velocidade de germinação, a partir de 25,0 ou 50,0 $\mathrm{gL}^{-1}$.

Tabela 3. Germinação (\%), índice de velocidade de germinação (IVG) e tempo médio de germinação (TMG) de sementes de tomate, $\mathrm{Ex}_{\mathrm{H} 2 \mathrm{O}}$ e $\mathrm{Ex}_{\mathrm{EtOH}}$, folhas de Pouteria ramiflora

\begin{tabular}{ccccccc}
\hline & \multicolumn{2}{c}{ Germinação $(\%)$} & \multicolumn{2}{c}{ TVG } & \multicolumn{2}{c}{ TMG } \\
\hline Extrato $\left(\mathrm{g} \mathrm{L}^{-1}\right)$ & $\mathrm{Ex}_{\mathrm{H} 2 \mathrm{O}}$ & $\mathrm{Ex}_{\mathrm{EtOH}}$ & $\mathrm{Ex}_{\mathrm{H} 2 \mathrm{O}}$ & $\mathrm{Ex}_{\mathrm{EtOH}}$ & $\mathrm{Ex}_{\mathrm{H} 2 \mathrm{O}}$ & $\mathrm{Ex}_{\mathrm{EtOH}}$ \\
\hline 0 & $96 \mathrm{a}$ & $96 \mathrm{a}$ & $13,2 \mathrm{a}$ & $13,2 \mathrm{a}$ & $1,2 \mathrm{~b}$ & $1,2 \mathrm{a}$ \\
25,0 & $97 \mathrm{a}$ & $95 \mathrm{a}$ & $12,5 \mathrm{a}$ & $11,5 \mathrm{~b}$ & $1,2 \mathrm{~b}$ & $1,2 \mathrm{a}$ \\
50,0 & $97 \mathrm{a}$ & $94 \mathrm{a}$ & $11,3 \mathrm{~b}$ & $11,7 \mathrm{~b}$ & $1,2 \mathrm{~b}$ & $1,2 \mathrm{a}$ \\
100,0 & $86 \mathrm{~b}$ & $95 \mathrm{a}$ & $7,3 \mathrm{c}$ & $11,6 \mathrm{~b}$ & $1,1 \mathrm{~b}$ & $1,3 \mathrm{a}$ \\
200,0 & $73 \mathrm{c}$ & $88 \mathrm{~b}$ & $2,3 \mathrm{~d}$ & $11,3 \mathrm{~b}$ & $0,5 \mathrm{a}$ & $1,2 \mathrm{a}$ \\
\hline
\end{tabular}

* Médias seguidas pelas mesmas letras dentro das colunas não diferem entre si, pelo teste t ( $5 \%$ de probabilidade).

O crescimento das plântulas, em câmara de germinação, foi afetado negativamente, em ambos os extratos, com as raízes sofrendo maior interferência negativa, a partir de 25,0 ou 50,0 gL $\mathrm{L}^{-1}$, alface (L. sativa) e tomate (L. esculentum). A parte aérea foi afetada apenas nas maiores concentrações, acima de 50,0 gL-1 (Tabela 4).

Tabela 4. Valores médios do comprimento $(\mathrm{mm})$ das raízes e caules de plântulas de tomate e alface, Ex $\mathbf{x}_{\mathrm{H} 2 \mathrm{O}} \mathrm{e}$ $\mathrm{Ex}_{\mathrm{EtOH}}$, em câmara de germinação, com folhas de Pouteria ramiflora

\begin{tabular}{ccccc|cccc}
\hline & \multicolumn{2}{c}{ Tomate } & \multicolumn{4}{c}{ Alface } \\
\hline \multirow{2}{*}{ Extrato $\left(\mathrm{g} \mathrm{L}^{-1}\right)$} & \multicolumn{2}{c}{$\mathrm{Ex}_{\mathrm{H} 2 \mathrm{O}}$} & \multicolumn{2}{c|}{$\mathrm{Ex}_{\mathrm{EtOH}}$} & \multicolumn{2}{c}{$\mathrm{Ex}_{\mathrm{H} 2 \mathrm{O}}$} & \multicolumn{2}{c}{$\mathrm{Ex}_{\mathrm{EtOH}}$} \\
\hline & Raiz & Caule & Raiz & Caule & Raiz & Caule & Raiz & Caule \\
\hline 0 & $13,7 \mathrm{a}$ & $21,2 \mathrm{a}$ & $11 \mathrm{a}$ & $20,1 \mathrm{a}$ & $13,4 \mathrm{a}$ & $18,1 \mathrm{a}$ & $16,1 \mathrm{a}$ & $7,7 \mathrm{a}$ \\
25,0 & $12,8 \mathrm{~b}$ & $22,3 \mathrm{a}$ & $7,5 \mathrm{~b}$ & $20,6 \mathrm{a}$ & $13,7 \mathrm{a}$ & $18,1 \mathrm{a}$ & $14,1 \mathrm{~b}$ & $8 \mathrm{a}$ \\
50,0 & $12,7 \mathrm{~b}$ & $21,9 \mathrm{a}$ & $7,1 \mathrm{~b}$ & $15,3 \mathrm{~b}$ & $11,4 \mathrm{~b}$ & $18,8 \mathrm{a}$ & $14,3 \mathrm{~b}$ & $7,6 \mathrm{a}$ \\
100,0 & $12,2 \mathrm{~b}$ & $16,1 \mathrm{~b}$ & $7,4 \mathrm{~b}$ & $14,6 \mathrm{~b}$ & $6,2 \mathrm{c}$ & $18,1 \mathrm{a}$ & $13,8 \mathrm{~b}$ & $7 \mathrm{~b}$ \\
200,0 & $6,6 \mathrm{c}$ & $16,2 \mathrm{~b}$ & $6,6 \mathrm{c}$ & $14,9 \mathrm{~b}$ & $3,6 \mathrm{~d}$ & $6,1 \mathrm{~b}$ & $8,6 \mathrm{c}$ & $7 \mathrm{~b}$ \\
\hline
\end{tabular}

* Médias seguidas pelas mesmas letras dentro das colunas não diferem entre si, pelo teste t ( $5 \%$ de probabilidade).

Em casa de vegetação (Tabela 5), a emergência das sementes de alface e tomate, nas diferentes concentrações, foi afetada negativamente; porém apenas o crescimento do sistema radicular de ambas as espécies foi reduzido. A parte aérea cresceu normalmente, em comparação com a testemunha. 
Tabela 5. Emergência (\%), índice de velocidade de emergência e comprimento (mm) das raízes e caules de plântulas de alface e tomate, controle e concentrações de 100 e $200 \mathrm{~g} \mathrm{~L}^{-1}$, em casa de vegetação, substrato com pó de folhas de Pouteria ramiflora

\begin{tabular}{|c|c|c|c|c|}
\hline Alface & Emergência (\%) & IVE & \multicolumn{2}{|c|}{ Comprimento } \\
\hline Concentração do extrato $\left(\mathrm{g} \mathrm{L}^{-1}\right)$ & & & Raiz & Caule \\
\hline 0 & $86,7 \mathrm{a}$ & $3,4 \mathrm{a}$ & $39,8 \mathrm{a}$ & $7,5 \mathrm{a}$ \\
\hline 100 & $55 \mathrm{~b}$ & $1,8 \mathrm{~b}$ & $22,1 \mathrm{~b}$ & $8,7 \mathrm{a}$ \\
\hline 200 & $21,7 \mathrm{c}$ & $0,3 \mathrm{c}$ & $22,1 \mathrm{~b}$ & $7,3 \mathrm{a}$ \\
\hline Tomate & Emergência $(\%)$ & IVE & \multicolumn{2}{|c|}{ Comprimento } \\
\hline Concentração do extrato $\left(\mathrm{g} \mathrm{L}^{-1}\right)$ & & & Raiz & Caule \\
\hline 0 & $61,7 \mathrm{a}$ & $1,8 \mathrm{a}$ & $57,4 \mathrm{a}$ & $37,9 \mathrm{a}$ \\
\hline 100 & $46,7 \mathrm{~b}$ & $1,3 \mathrm{a}$ & $46,1 \mathrm{~b}$ & $36,4 \mathrm{a}$ \\
\hline 200 & $21,7 \mathrm{c}$ & $0,4 \mathrm{~b}$ & $31,7 \mathrm{c}$ & $32,4 \mathrm{a}$ \\
\hline
\end{tabular}

* Médias seguidas pelas mesmas letras dentro das colunas não diferem entre si, pelo teste t ( $5 \%$ de probabilidade).

Avaliando-se as taxas de germinação e crescimento, associados aos resultados qualitativos e quantitativos da análise química, com exceção dos açúcares redutores e saponinas, que existem em maior concentração em $\mathrm{Ex}_{\mathrm{H} 2 \mathrm{O}}$ os demais metabólitos são encontrados em maior número e concentração em $\mathrm{Ex}_{\mathrm{EtOH}}$; apesar desta maior concentração, este extrato teve menor efeito sobre a germinação das espécies-alvo. Por outro lado, em $\operatorname{Ex}_{\mathrm{H} 2 \mathrm{O}} \mathrm{O}$ sinergismo dos metabolitos e a presença de saponinas foram mais eficazes em afetar negativamente as espécies testadas.

Também pode ser observado que os efeitos fitotóxicos foram mais intensos para o sistema radicular das duas espécies analisadas, tanto para o crescimento em câmara de germinação como em casa de vegetação e à medida que a concentração dos extratos aumentava, o tamanho das plântulas era afetado mais intensamente.

Esta ação pode estar relacionada com a absorção e, consequentemente, com a concentração de fitotoxinas nos tecidos radiculares, situação favorecida pelo contato físico da raiz com o substrato contendo $\mathrm{Ex}_{\mathrm{H} 2 \mathrm{O}}$ e $\mathrm{Ex}_{\mathrm{EtOH}}$. Segundo Correia et al. (2005), a bioatividade dos extratos está condicionada à capacidade de absorção, translocação e mecanismos de ação das substâncias potencialmente alelopáticas e sua ação pode diferir, na dependência da espécie.

Os resultados apresentados confirmam a afirmação de Ferreira e Áquila (2000), onde o efeito alelopático normalmente é mais intenso sobre o crescimento do que sobre a germinação, o que pode ser observado no presente trabalho. Porém as alterações no padrão de desenvolvimento podem ser resultado de inúmeros efeitos causados a nível primário. Os mesmos autores enfatizam as alterações na permeabilidade de membranas, no funcionamento de mensageiros secundários, na respiração, na conformação de enzimas e receptores, ou ainda, pela combinação destes e outros fatores, o que pode afetar diretamente o crescimento das plântulas.

Esta redução no desenvolvimento pode ser observada no trabalho de Maia et al. (2011), analisando o efeito do solo coletado em área cultivada anteriormente com hortelã e posteriormente com alface, que apresentou diminuição de plântulas emergidas. De acordo com Cochran et al. (1977), os compostos lixiviados no solo, resultantes da decomposição dos vegetais, demonstraram afetar negativamente o desenvolvimento e produtividade de diferentes culturas, o que está de acordo como os resultados encontrados neste estudo com $P$. ramiflora.

\section{Conclusão}

Em condições laboratoriais e de casa de vegetação, os extratos de folhas de $P$. ramiflora apresentam potencial alelopático sobre a germinação de cipselas de alface (L. sativa) e sementes de tomate (L. esculentum) e posterior formação de plântulas, interferindo significativamente no vigor das sementes das espécies-alvo, principalmente 
no desenvolvimento da raiz principal. Este efeito pode estar associado, de forma geral, a presença de compostos fenólicos, taninos, flavonoides, cumarinas e saponinas.

Considerando os dados químicos obtidos, é evidente que a espécie em estudo é uma fonte rica de compostos fenólicos, principalmente os flavonoides com expressiva atividade antioxidante, com grande potencial econômico, para obtenção de metabólitos secundários e fonte de pesquisa para possíveis herbicidas naturais. Porém sua utilização em áreas degradadas, visando sua recuperação, deve ser feita de maneira criteriosa devido a sua capacidade de interferir no desenvolvimento de outras espécies.

\section{Agradecimentos}

À CAPES, pelas bolsas de mestrado e doutorado e ao CNPq, pelas bolsas de Produtividade em Pesquisa e Iniciação Cientifica (PIBIC), concedidas. E o apoio financeiro do CNPq, CPP, INAU, FUNDECT e a Universidade pelo financiamento do Grupo Interdisciplinar de Pesquisa (GIP) e de Produtos Naturais (PN).

\section{REFERÊNCIAS}

Abenavoli MR, Cacco G, Sorgonà A, Marabottini R, Paolacci AR, Ciaffei M and Badiami M. 2006. The inhibitory effects of coumarin on the germination of durum wheat (Triticum turgidum ssp. durum, cv. Simeto) seeds. Journal of Chemical Ecology, 32(2):489-506.

Capobiango RA, Vestena S e Bittencourt HC. 2009. Alelopatia de Joanesia princeps Vell. e Casearia syllvestris Sw. sobre espécies cultivadas. Revista Brasileira de Farmacognosia, 9(4): 924-930.

Cochran VL, Elliott LF and Papendick RI. 1977. The production of phytotoxins from surface crop residues. Soil Science Society of America Journal, 41(5):903-908.

Condessa MC, Carvalho IAF, Santos BTA, Paula JE, Gomes SM, Silveira D e Simeoni LA. 2013. Efeito de extratos foliares de três espécies de Pouteria (Sapotaceae) sobre a germinação e crescimento de Lactuca sativa L. e Lycopersicum esculentum Mill. Revista Brasileira de Biologia e Farmácia, 9(3):1-18.

Correia NM, Centurion MAPC e Alves PLCA. 2005. Influência de extratos aquosos sobre a germinação e o desenvolvimento de plântulas de soja. Ciência Rural, 35(3):498-503.

Costa AF. Farmacognosia. 2002. v.1. 5ed. Lisboa: Fundação Calouste Gulbenkian. 604 p.

Costa Neto POL. Estatística. São Paulo: Edgard Blücher, 2002. 264 p.

Dias DCFS e Marcos Filho J. 1995. Testes de vigor baseado na permeabilidade das membranas celulares. I. Condutividade elétrica. Informativo ABRATES, 5:26-36.

Fernandes RM, Oliveira AKM, Abreu CAA e Silva BCFL. 2014. Ação alelopática dos galhos de Pouteria ramiflora (Mart.) Radlke na germinação de Lactuca sativa (alface) e Lycopersicon esculentum (tomate). Cadernos de Agroecologia, 9(4):1-4.

Ferreira AG e Áquila MEA. 2000. Alelopatia: uma área emergente da ecofisiologia. Revista Brasileira de Fisiologia Vegetal, 12(edição especial):175-204.

Ferreira DF. Estatística básica. Lavras: UFLA, 2005. 625 p. 
Fontoura FM, Matias R, Ludwig J, Oliveira AKM, Bono JAM, Martins PDFRB e Guedes NMR. 2015. Seasonal effects and antifungal activity from bark chemical constituents of Sterculia apetala (Malvaceae) at Pantanal of Miranda, Mato Grosso do Sul, Brazil. Acta Amazonica, 45(3):283-292.

Fujii Y and Hiradate S. 2007. Allelopathy: new concepts \& methodology. Enfield: Science Publishers. 382 p.

Lorenzi H. 2002. Árvores Brasileiras: manual de identificação e cultivo de plantas arbóreas do Brasil. v.1. 4ed. Nova Odessa: Instituto Plantarum. 368 p.

Maguire JD. Speed of germination-aid in selection evaluation for seedling emergence and vigour. 1962. Crop Science, 2(2):176-177.

Maia JTLS, Bonfim FPG, Barbosa CKR, Guilherme DO, Honório ICG e Martins ER. 2011. Influência alelopática de hortelã (Menta x villosa Huds.) sob emergência de plântulas de alface (Lactuca sativa). Revista Brasileira de Plantas Medicinais, 13(3):253-257.

Martins CC, Bovi MLA e Spiering SH. 2009. Umedecimento do substrato na emergência e vigor de plântulas de pupunheira. Revista Brasileira de Fruticultura, 31(1):224-230.

Matos JFA. 2010. Introdução a fitoquímica experimental. 3ed. Fortaleza: UFC. 141 p.

Montenegro LHM, Oliveira PES, Conserva LM, Rocha EMM, Brito AC, Araújo RM, Trevisan MTS e Lemos RPL. 2006. Triterpenóides e avaliação do potencial antimalárico, larvicida, anti-radicalar e anticolinesterástico de Pouteria venonosa (Sapotaceae). Revista Brasileira de Farmacognosia, 16(suplemento):611-617.

Nemoto LRP. 2005. Plantas daninhas na cultura da soja. Boletim de Pesquisa de Soja. Rondonópolis: Fundação Mato Grosso. 95 p.

Nicollier GF and Thompson AC. 1982. Phytotoxic compounds from Melilotus alba (white sweet clover) and isolation and identification of two new flavonoids. Journal of Agricultural and Food Chemistry, 30(4):760-764.

Oliveira AKM, Ribeiro JWF, Matias R, Gusmão DH e Pereira KCL.2011. Potencial alelopático de folhas frescas de bacupari (Rheedia brasiliensis (Mart.) Planch. \& Triana) na germinação de alface. Revista Brasileira de Biociências, 9(4):550-553.

Oliveira AKM, Pereira KCL, Muller JAI e Matias R. 2014a. Análise fitoquímica e potencial alelopático das cascas de Pouteria ramiflora na germinação de alface. Horticultura Brasileira, 32(1):41-47.

Oliveira AKM, Matias R, Lopes SS e Fontoura MF. 2014b. Alelopatia e a influência de folhas de Palicourea rigida (Rubiaceae) na germinação de sementes e na formação de plântulas de alface. BioScience Journal, 30(supplement)2:938-947.

Parvez MM, Tomita-Yokotani K, Fujii Y, Konishi T and Iwashina T. 2004. Effects of quercetin and its seven derivatives on the growth of Arabidopsis thaliana and Neurospora crassa. Biochemical Systematics and Ecology, 32(7):631-635.

Pedro N, González L and Reigosa MJ. 2006. Allelopathy and abiotic stress. In: Reigosa MJ, Pedro N and González L (Editors). Allelopathy: a physiological process with ecological implications. Dordrecht: Springer.p.171-209.

Peixoto Sobrinho TJS, Silva CHTP, Nascimento JE, Monteiro JM, Albuquerque UP e Amorim ELC. 2008. Validação de metodologia espectrofotométrica para qualificação dos flavonoides de Baubinia cheilantha (Bongard) Steudel. Revista Brasileira de Ciências Farmacêuticas, 44:683-689. 
Pergo EM and Ishii-Iwamoto EL. 2011. Changes in energy metabolism and antioxidant defense systems during seed germination of the weed species Ipomoea triloba L. and the responses to allelochemicals. Journal of Chemical Ecology, 37(5):500-514.

Rice EL. 1984. Allelopathy. New York: Academic Press. 422 p.

Sausen TL, Löwe TR, Figueiredo LS e Buzatto CR.2009. Avaliação da atividade alelopática do extrato aquoso de folhas de Eugenia involucrata DC. e Acca sellowiana (O. Berg) Burret. Polibotánica, 27:145-158.

Silva GB, Martim L, Silva CL, Young MCM e LadeiraAM. 2006. Potencial alelopático de espécies arbóreas nativas do Cerrado. Hoehnea, 33: 331-338.

Silva CAM, Simeoni LA and Silveira D. 2009. Genus Pouteria: chemistry and biological activity. Revista Brasileira de Farmacognosia, 9(2):501-509.

Simões CMO, Schenkel EP, Gosmann G, Mello JCP, Mentz LA e Petrovick PR. 2004. Farmacognosia, da planta ao medicamento. 5ed Florianópolis: UFRGS. 1002 p.

Snedecor GW and Cochram WG. Statistical methods. Ames: Iowa State University, 1973. 593 p.

Sousa CM, Rocha e Silva H, Vieira-Jr GM, Ayres MCC, Costa CLS, Araújo DS, Cavalcante LCD, Barros EDS, Araújo PBM, Brandão MS e Chaves MH. 2007. Fenóis totais e atividade antioxidante de cinco plantas medicinais. Química Nova, 30:351355.

Souza CLM, Morais V, Silva ER, Lopes HM, Tozani R, Parraga MS e Carvalho GA. 1999. Efeito inibidor dos extratos hidroalcóolicos de coberturas mortas sobre a germinação de sementes de cenoura e alface. Planta Daninha, 17(2):263-271.

Souza SAM, Cattelan LV, Vargas DP, Piana CFB, Bobrowski VL e Rocha BHG. 2005. Efeito de extratos aquosos de plantas medicinais nativas do Rio Grande do Sul sobre a germinação de sementes de alface. Publicatio UEPG: Ciências Biológicas e da Saúde, 11:29-38.

Thaipong K, Boonprakoba U, Crosbyb K, Cisneros-Zevallosc L and Byrnec DH. 2006. Comparison of ABTS, DPPH, FRAP, and ORAC assays for estimating antioxidant activity from guava fruit extracts. Journal of Food Composition and Analysis, 19:669-675.

Vieira RD e Carvalho NM. 1994. Testes de vigor em sementes. Jaboticabal: FUNEP. 164 p.

Wandscheer ACD e Pastorini LH. 2008. Interferência alelopática de Raphanus raphanistrum L. sobre a germinação de Lactuca sativa L. e Solanum lycopersicon L. Ciência Rural, 38(4):949-953.

Weir T, Park SW and Vivanco JM. 2004. Biochemical and physiological mechanisms mediated by allelochemicals. Current Opinion in Plant Biology, 7:472-479.

Willis RJ. 2007. The history of allelopathy. Dordrecht: Springer. 316 p.

Zeng RS, Mallik AU and Luo S. 2010. Allelopathy in sustaintable agriculture and forestry. New York: Springer-Verlag. $426 \mathrm{p}$. 